\title{
Untersuchungen zur Abschätzung von Messunsicherheiten mit dem virtuellen Rasterelektronenmikroskop vREM
}

\author{
Dominic Gnieser ${ }^{1,2}$, Carl Georg Frase ${ }^{2}$, Klaus-Peter Johnsen ${ }^{2}$, Rainer Tutsch ${ }^{1}$, Harald Bosse ${ }^{2}$ \\ 1: Institut für Produktionsmeßtechnik, Technische Universität Braunschweig, \\ Schleinitzstraße 20,38106 Braunschweig \\ 2: Physikalisch-Technische Bundesanstalt, \\ Bundesallee 100, 38116 Braunschweig
}

Tel.-Nr.: 0531-391-7025, E-Mail-Adresse(n): D.Gnieser@tu-braunschweig.de, Carl.G.Frase@ptb.de, Klaus-Peter.Johnsen@ptb.de, R.Tutsch@tu-braunschweig.de, Harald.Bosse@ptb.de

\section{Zusammenfassung}

Der aktuelle Status des virtuelles Rasterelektronenmikroskop vREM, ein Programm entwickelt in Zusammenarbeit des Instituts für Produktionsmesstechnik (IPROM) der technischen Universität Braunschweig mit der Physikalisch-Technischen Bundesanstalt (PTB) wird vorgestellt. Das Programm hat zum Ziel, den gesamten Messvorgang eines Rasterelektronenmikroskops zu simulieren, und die auftretenden Messunsicherheiten zu bestimmen.

Der Aufbau und die Funktionsweise des Programms werden dargestellt: Die Module Elektronenquelle, Elektronenoptik, Rastergenerator, Probengeometrie und Wechselwirkung, Detektion und Bildgenerierung sowie Auswertung werden erläutert, die maßgeblichen Parameter und deren Wirkungsweise vorgestellt.

\section{Einleitung}

Um ein Messverfahren metrologisch rückführbar zu machen, ist es notwendig, eine auf die jeweilige spezielle Messaufgabe zugeschnittene Bestimmung der Messunsicherheit durchzuführen. Bei komplexen Systemen ist ein Ansatz zur Abschätzung der Messunsicherheit das so genannte virtuelle Messgerät: Im Computer wird der Messprozess mit seinen Einflussgrößen simuliert und über Monte-Carlo-Rechnungen wird eine statistische Analyse durchgeführt.

Solche computerbasierten Modellierungen, die die jeweiligen messtechnisch relevanten Eigenschaften virtuell nachbilden, gibt es bereits für viele Messsysteme der Mikro- und Nanotechnologie [1]. Ziel des Projekts, das hier vorgestellt wird, ist es, ein solches virtuelles Messgerät auch für das Rasterelektronenmikroskop (REM) zu erstellen und damit GUM-konforme Abschätzungen der Messunsicherheit für dimensionelle Messaufgaben im Bereich der Mikrosystemtechnik und Nanotechnologie geben zu können. Damit einhergehend werden modellbasierte Korrekturen von systematischen Abweichungen ermöglicht, Erkenntnisse über die Stärke verschiedener Störeinflüsse können auch zu Empfehlungen für reale Messungen führen. So können dominantere von eher nachgeordneten Unsicherheitsbeiträgen für spezifische Messaufgaben unterschieden werden, um Messbedingungen systematisch zu verbessern (Messplan-Design) und Komponentenoptimierungen anregen zu können.

Die Modellierung mit dem Namen „VREM“ beinhaltet alle wesentlichen Komponenten der Messkette eines REM als Module: Die Elektronenquelle, das elektronenoptische Linsensystem, den Rastergenerator, die Wechselwirkung der Elektronen mit dem Objekt, Detektoren, sowie einfache Auswerteprozeduren. Über Parameter einstellbar, können Unsicherheitsbeiträge zur virtuellen Probe, zum virtuellen Prüfling und zum virtuellen Detektor beigesteuert werden. Äußere Faktoren wie Temperatur, Vibrationen oder Streufelder beeinflussen dabei zusätzlich die jeweiligen Komponenten. Das virtuelle Messgerät vREM basiert dabei auf dem an der PTB entwickelten, in C++ geschriebenen MonteCarlo-Programm MCSem [2], das die Bildentstehung im REM simuliert und schwerpunktmäßig die physikalische Wechselwirkung zwischen den Probenelektronen und den Atomen des Untersuchungsobjektes behandelt. Eine Übersicht über den Programmablauf ist in Abbildung 1 zu sehen. 


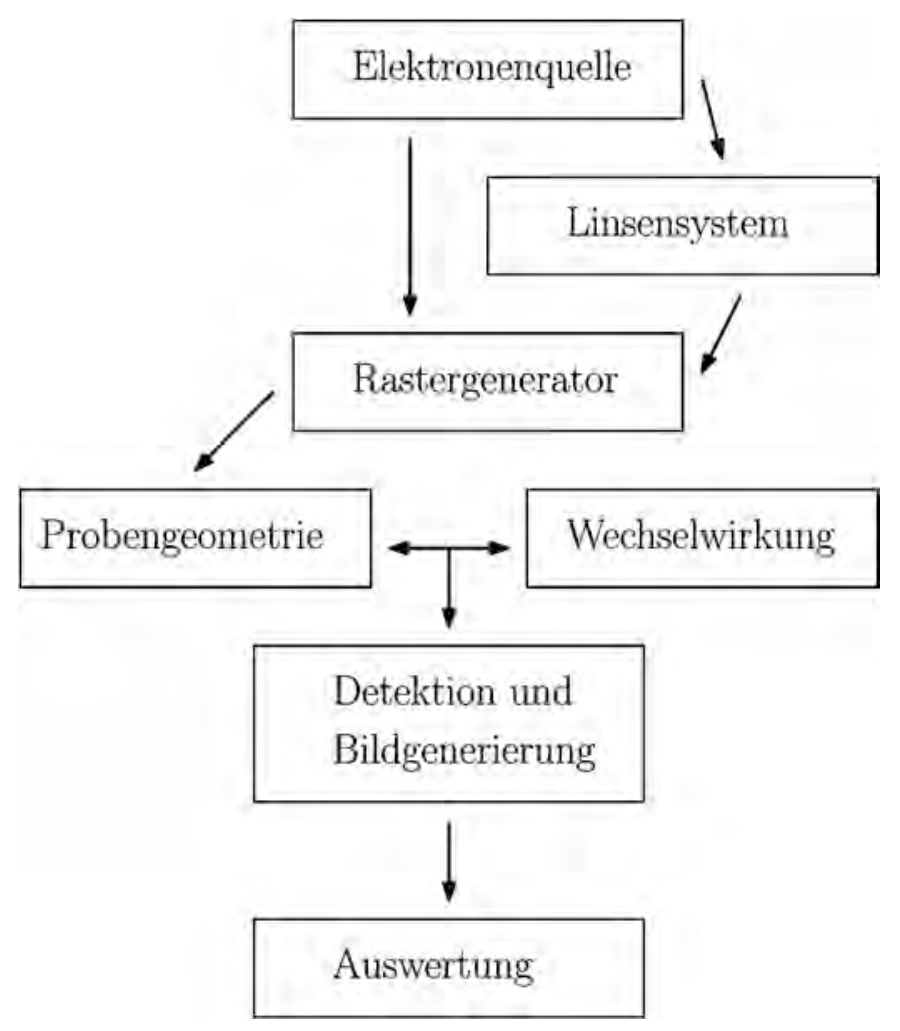

Abbildung 1: Modularer Aufbau des Programmes vREM

\section{Beschreibung des Programms vREM}

Um mit vREM Unsicherheiten für eine Messaufgabe abschätzen zu können sind zusätzlich zu den aufwendigen Monte-Carlo-Rechnungen der Bildentstehung gegebenenfalls viele verschiedene weitere Parameter zu variieren. Das ist mit einem stark erhöhten Rechenaufwand verbunden. Daher wurde als Programmiersprache MATLAB gewählt, welches stark optimierte Rechenroutinen zu Verfügung stellt und bei geeigneter Programmierung eine parallele Ausführung vieler Rechnungen ermöglicht, was die Dauer der Simulationsdurchläufe verkürzt. Auch stellt MATLAB eine Möglichkeit zur Erstellung von graphischen Benutzer-Interfaces (GUI) zu Verfügung, was eine spätere Nutzung von vREM für nichtspezialisierte Nutzer erleichtert. In Abbildung 2 oben rechts ist das momentane Hauptmenu von vREM zu sehen, von dem aus die verschiedenen Module zugänglich sind (das Auswertungs-Modul ist noch nicht integriert) und das die eingestellten Datensätze anzeigt. Über den "change-Button“ kommt man in das jeweilige Modul-Menu und kann dort andere Datensätze aus Dateien laden oder die Parameter von Hand verändern und abspeichern. Der „apply-Button“ führt Berechnungen mit dem aktiven Parametersatz durch.

\section{Das Modul der Elektronenquelle}

In Abbildung 2 links unten ist das Menu für das Modul der Elektronenquelle gezeigt. Über die dort angegebenen Parameter lassen sich eine große Anzahl von Variationen bei den Elektrodentypen durchführen. Die Parameter sind im einzelnen:

Der virtuelle Kathodendurchmesser beschreibt den Durchmesser des ersten Crossovers der Kathode, der auf die Probenoberfläche durch das Linsensystem verkleinert abgebildet wird. Bei Glühkathoden liegt dieser im Bereich von $20-50 \mu \mathrm{m}$, Schottky-Emitter und Feldemissionskathoden (FE) können virtuelle Durchmesser von unter $10 \mathrm{~nm}$ erzeugen. 
Über die Kathodentemperatur erhalten die Elektronen die nötige Energie, um die Austrittsarbeit zu bewältigen und aus der Kathode auszutreten. Bei Glühkathoden liegt die Temperatur je nach Material zwischen etwa $1500 \mathrm{~K}$ und $3000 \mathrm{~K}$ (bei Austrittsarbeiten zwischen 2,7eV und 4,5eV). Bei SchottkyEmittern wird die effektive Austrittsarbeit durch ein angelegtes Feld vermindert, diese können daher bei einer niedrigeren Temperatur betrieben werden. FE-Kathoden funktionieren durch die starken Felder bei Raumtemperatur, werden aber häufig im Bereich von 1000K - 1500K betrieben.

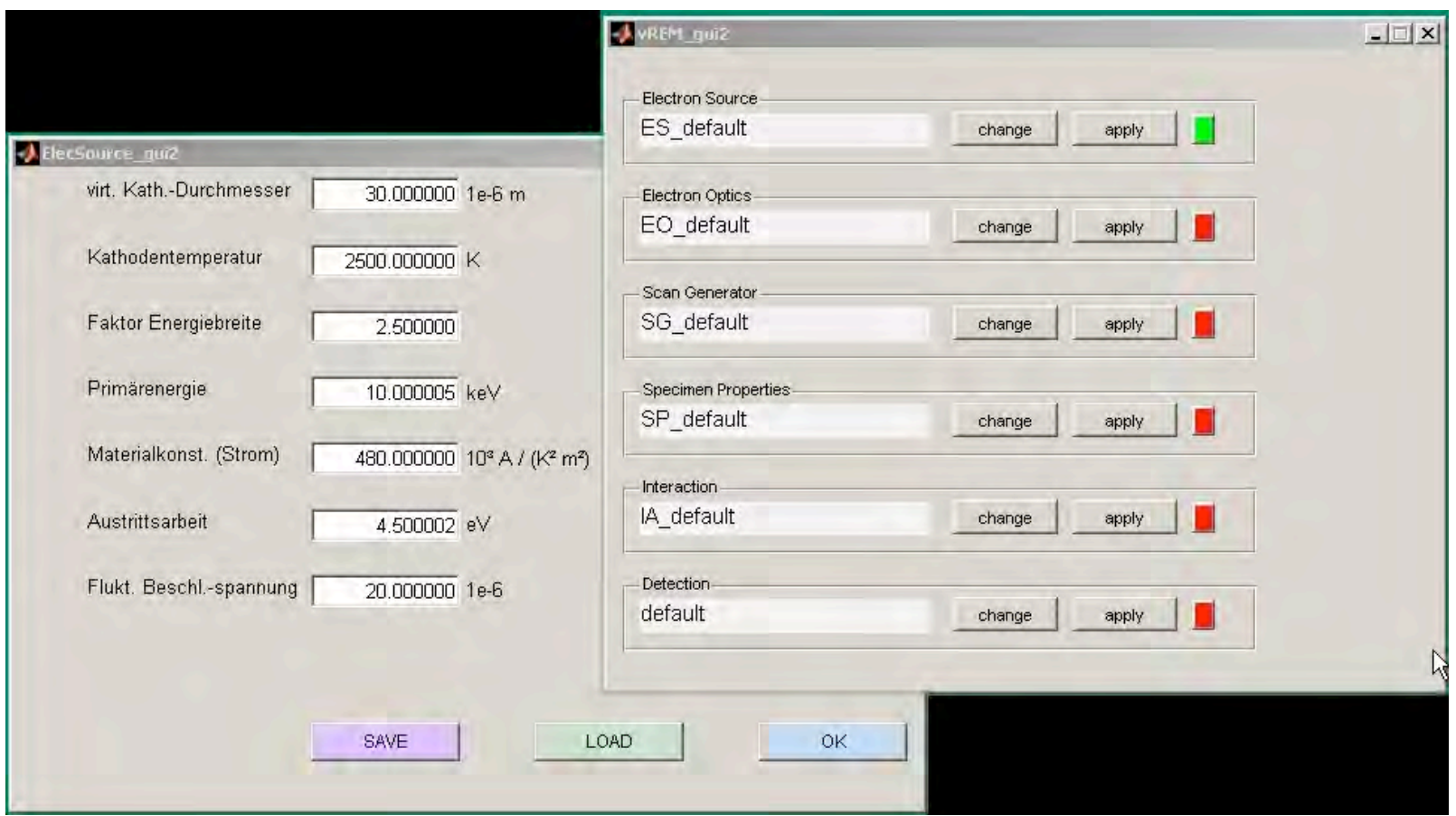

Abbildung 2: GUI des Programms vREM. Rechts oben ist der Haupt-Auswahlbildschirm mit den verschiedenen Modulen und den jeweiligen, momentan aktiven Datensätzen zu sehen. Links unten ist die GUI der Elektronenquelle mit seinen beschreibenden Parametern dargestellt.

Aus Temperatur und Austrittsarbeit kann man mit einer materialabhängigen Konstante über das Richardson-Gesetz die Strahlstromdichte bestimmen. Die Temperatur bestimmt zusammen mit dem Faktor für die Energiebreite den Streubereich der Energie, den die Primärelektronen haben (vereinfacht ohne den Boersch-Effekt simuliert).

Weitere Parameter zur Simulation der Kathode sind die vorgesehene Primärenergie auf die die Elektronen beschleunigt werden sollen, und die Fluktuationen $\Delta U / U$ dieser Beschleunigungsspannung. Aus Strahlstromdichte, Primärenergie und Temperatur lässt sich wiederum der maximale Richtstrahlwert berechnen, aus dem sich später mit dem Aperturwinkel der Probenstrom herleitet.

Die Formeln für diese Beziehungen und die Werte der Parameter für die verschiedenen Kathodentypen finden sich z.B. in [3].

\section{Das Modul der Elektronenoptik}

In Abbildung 3, unten links ist die Eingabemaske für die Parameter des Elektronenoptik-Moduls zu sehen. Durch dieses Modul soll es möglich sein, die wichtigsten Abbildungsfehler zu simulieren und zu variieren:

Der Durchmesser des Strahlflecks, der durch die sphärische Aberration entsteht, leitet sich aus dem zugehörigen Beiwert $C_{S}$ und dem Aperturwinkel $\alpha$ ab: $d_{s}=0,5 C_{s} \alpha^{3}$

Der Strahlfleck der chromatischen Aberration wird bestimmt aus dem entsprechenden Beiwert $\mathrm{C}_{\mathrm{C}}$, dem Aperturwinkel $\alpha$ und einer Kombination aus den schon im Kathoden-Modul bestimmen Schwankung der 
Beschleunigungsspannung, dem Verhältnis der Energiebreite zur Primärenergie und der in diesem Modul angegeben Fluktuation der Versorgungsströme der magnetischen Linsen zusammen:

$\mathrm{d}_{\mathrm{C}}=\mathrm{C}_{\mathrm{C}} \Delta \mathrm{f} / \mathrm{f} \alpha$, wobei $\Delta \mathrm{f} / \mathrm{f}=\operatorname{sqrt}\left((\Delta \mathrm{U} / \mathrm{U})^{2}+(2 \Delta \mathrm{l} / \mathrm{l})^{2}+(\Delta \mathrm{E} / \mathrm{E})^{2}\right)$

Die Strahlfleckverbreiterung durch Astigmatismus ist proportional zum Abstand $\Delta f_{A}$ zwischen der sagittalen und der meridionalen Fokuslinie und dem Aperturwinkel: $d_{A}=\Delta f_{A} \alpha$

Die Strahlverbreiterung durch Beugung leitet sich aus dem Fraunhofer-Beugungsmuster (AiryScheibchen) ab und ist proportional zu der de Broglie Wellenlänge $\lambda$ des jeweiligen Elektrons (abhängig von der Energie), und umgekehrt proportional zum Aperturwinkel. Die Sinc-Funktion hat eine Halbweite von $d_{B}=0,61 \lambda / \alpha$

Für jedes Elektron wird auf die Verteilung der jeweiligen Verbreiterungen gewürfelt, hinzu kommt die durch die Linsen verkleinerte Verbreiterung des virtuellen Kathodendurchmessers. (Der Verkleinerungsfaktor lässt sich in der beschriebenen Version von vREM noch nicht als Parameter einstellen, sondern nur im Programmcode).

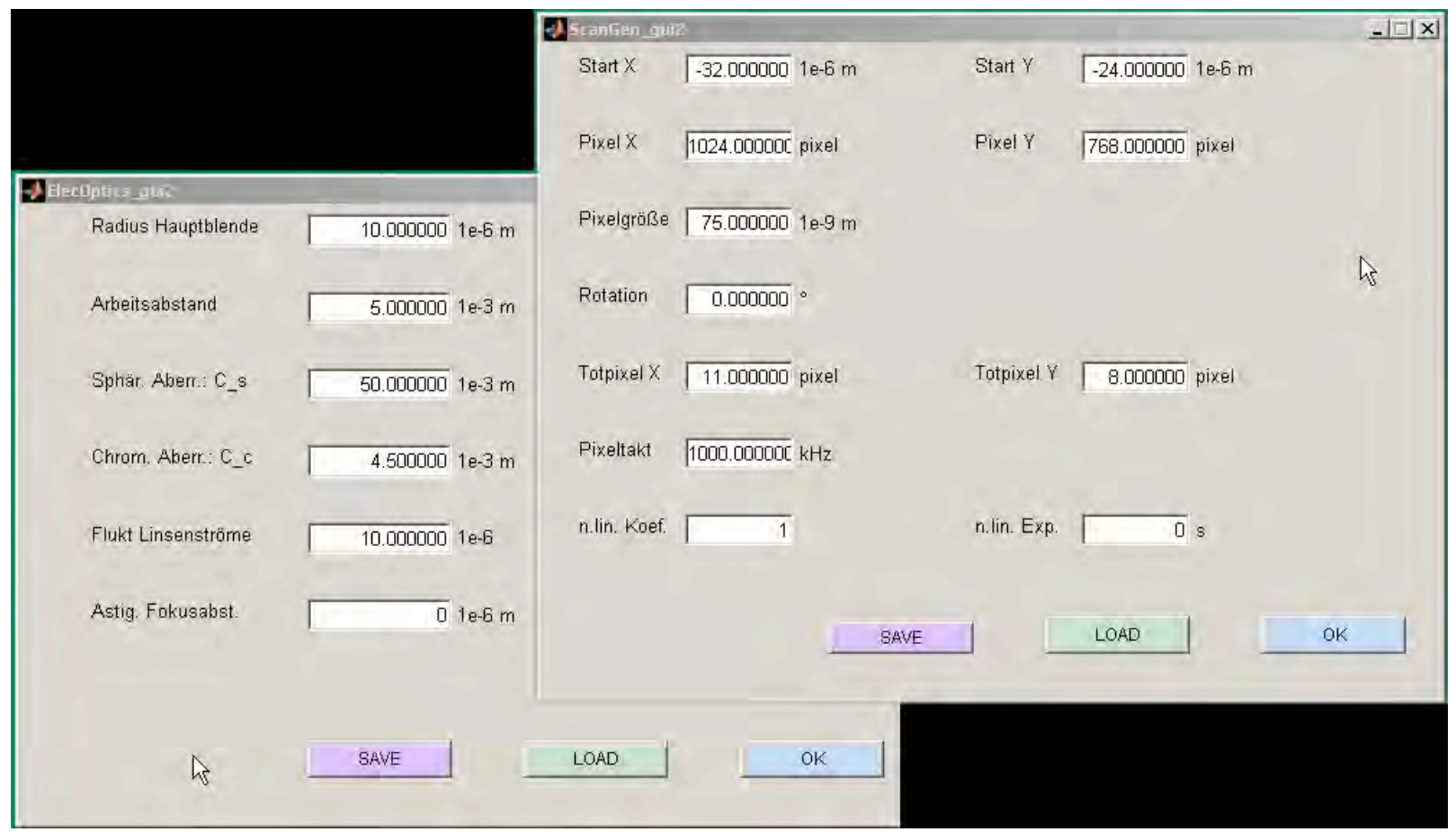

Abbildung 3: GUI des Programms vREM. Links unten sieht man die GUI des elektronenoptischen Moduls mit einem beispielhaften Parameter-Datensatz. Rechts oben ist das Fenster für das Rastergenerator-Modul abgebildet.

Der Aperturwinkel $\alpha$, wird berechnet aus dem Arcustangens des Verhältnisses des Radius der Hauptapertur zum Arbeitsabstand. Wobei darauf zu achten ist, dass sich die Hauptblende oberhalb der Objektiv-Linse befinden kann, in dem Fall ist der Radius der Abbildung der Hauptblende durch die Linse als Parameter zu verwenden.

In Abbildung 4 sind simulierte Daten für den mittleren Radius des Strahlflecks für verschiedene Arbeitsabstände (und damit unterschiedliche Aperturwinkel) aufgetragen. Die übrigen Werte sind die, die in Abb. 2 und Abb. 3 bei den jeweiligen Parametern eingetragen sind. Die unterste Kurve (Rauten) stellt nur die sphärische Aberration da, die mittlere Kurve (Quadrate) beinhaltet sphärische und chromatische Aberration, die obere Kurve (Dreiecke) dann zusätzlich auch die Beugungsverbreiterung (zur Vereinfachung nicht als sinc-Verteilung oder Gauß-Verteilung, sondern als Gleichverteilung angenommen). Da der astigmatische Fokusabstand auf null gesetzt wurde, ist keine AstigmatismusVerbreiterung vorhanden. Man sieht auch, dass der chromatische Anteil bei der simulierten Primärenergie von $10 \mathrm{keV}$ kaum eine Rolle spielt. Bei großen Arbeitsabständen (und damit kleinen 
Aperturwinkeln) dominiert der Beugungsanteil, bei kleinen Arbeitsabständen (großen Aperturwinkeln) ist die sphärische Aberration ausschlaggebend.

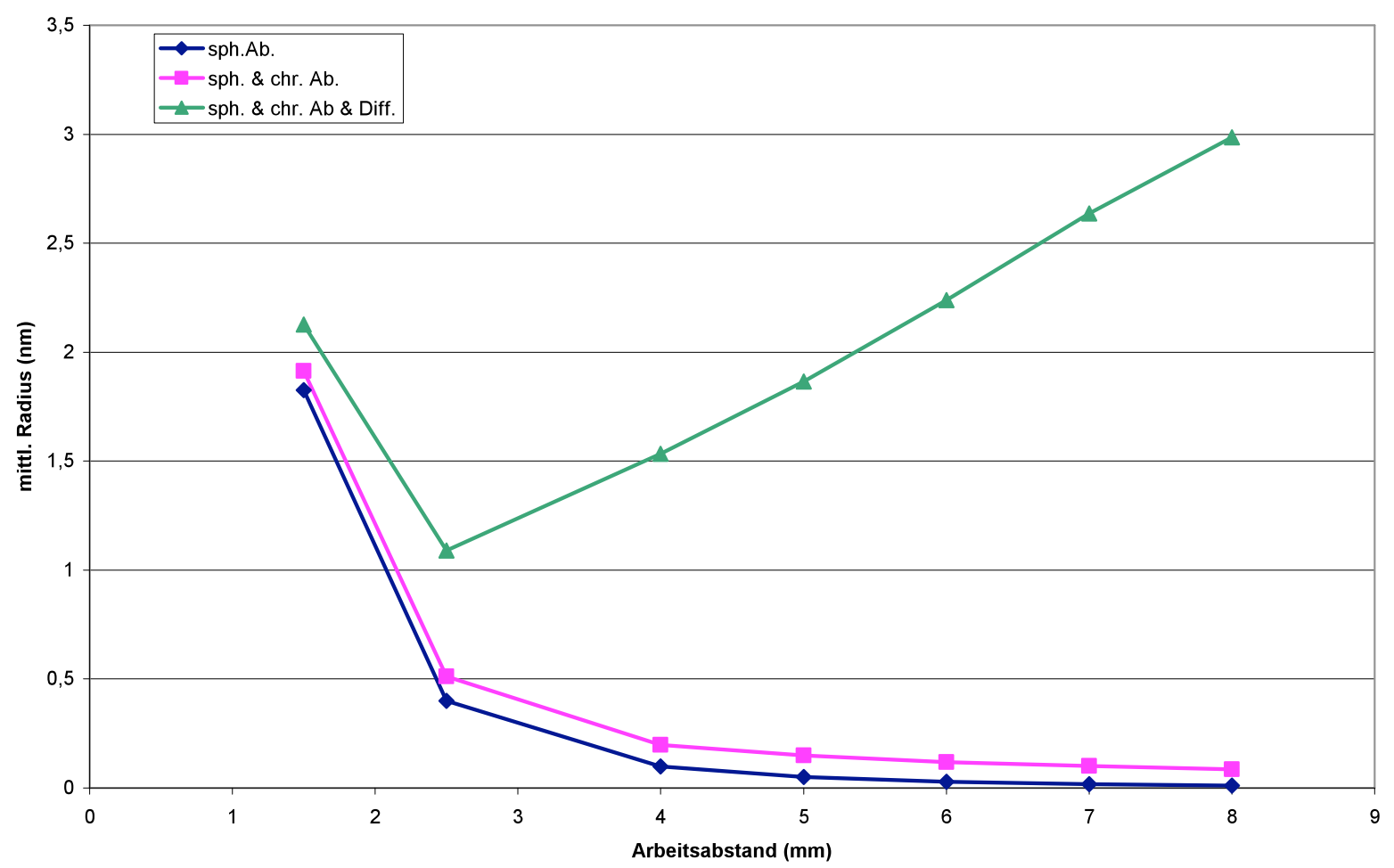

Abbildung 4: Der Einfluss der verschiedenen Aberrationen auf die Strahlfleckgröße, abhängig vom Arbeitsabstand.

\section{Das Rastergenerator-Modul}

In Abbildung 3 ist rechts oben das Fenster für das Modul des Rastergenerators zu sehen. StartX und StartY geben die Koordinaten (im Koordinatensystem in dem auch das Untersuchungsobjekt beschrieben wird) an, bei denen der Elektronenstrahl mit der Untersuchung beginnen soll. Darunter ist die Größe des Rasterfeldes in Pixel, sowie die Pixelgröße definiert. Der Wert Rotation wird benötigt, wenn die ScanRichtung nicht entlang der Achsen der Rasterablenkspulen (Schnelle Richtung: x. Langsame Richtung: y) erfolgen soll, sondern in einem Winkel dazu. Die angegebenen „Totpixel“ sind zusätzliche Pixel, die der Elektronenstrahl zwar abrastert, aber die eigentlich nicht im sichtbaren Bild vom Detektor angezeigt werden sollen. Der angegebene Pixeltakt gibt die Geschwindigkeit an, mit der Elektronenstrahl die Probe abrastert. Die beiden übrigen Parameter sind für die Steuerung der Scan-Nichtlinearitäten (wie z.B. gemessen in [4]) zuständig die sich aus der Reluktanz der Rasterspulen ergeben. Es wird eine Funktion genutzt, die sich am Einschaltvorgang bei Spulen orientiert:

$A(1-\exp (-t / T))$

Ein maßgeblicher Unterschied zum Ansatz in MCSem ist, dass die Zuordnung der eingestrahlten Elektronen nicht mehr nach der eigentlich "beabsichtigten" Pixelkoordinate erfolgt, sondern dass jedes Elektron nun einen Zeitindex - berechnet aus dem Strahlstrom, dem Pixeltakt und der Anzahl der zu scannenden Pixel (und Totpixel) - bekommt, der in dem späteren Detektormodul dann über Aufnahmezeitintervalle einem Bildpixel zugeordnet wird.

Im Anschluss an dieses Modul wird jedes Elektron mittels der in den bisherigen Programmteilen bestimmten Parameter und Verteilungen (sowie erwürfelten Zufallszahlen) durch einen Zustandsvariablensatz, bestehend aus Orts-Koordinaten (x, y, z), Richtungswinkeln $(\theta, \varphi)$, Energiewert und Zeitindex identifiziert sein.

An dieser Stelle werden dann auch Relativbewegungen zwischen der zu untersuchenden Struktur und dem scannenden Strahl, z.B. durch eingekoppelte Schwingungen, realisiert. 


\section{Probenwechselwirkung und Probengeometrie}

Die Wechselwirkung der Elektronen mit dem Festkörpermaterial der Probe und das Modul der Probengeometrie, dass dem Wechselwirkungsteil Materialdaten abhängig von der Position bereitstellt, wurden von dem in $\mathrm{C}++$ geschriebenen Programm MCSem in MATLAB übertragen und in die vREMStruktur eingegliedert. Dabei wurde die in $\mathrm{C}++$ auf Schleifen basierenden Simulationsabfolgen möglichst ideal an die auf Matrizen und Vektoren basierenden parallelen Beschreibungen MATLABs übertragen um möglichst hohe Berechnungsgeschwindigkeiten zu erreichen.

Im 3D-Geometriemodul lassen sich Strukturen frei wählbar aus einer Gruppe von Basiskörpern wie Quader, Ellipsoide, Zylinder, Schichten oder Dreiecksprismen zusammenstellen, wobei jeder Basiskörper aus einem individuelles Material besteht. Das Material kann ein einzelnes Element oder ein Komposit aus mehreren Elementen sein (oder Vakuum).

Das Wechselwirkungsmodul simuliert die Elektronenbewegung im Festkörper. Die elastische Streuung der Primärelektronen wird über tabellierte Mott-Streuquerschnitte berechnet, die mit einer modifizierten Version des Programms PWADIR von Salvat und Mayol [5] erstellt wurden. Die inelastische Streuung wird modelliert durch Bethes continuous slowing down approximation (CSDA) [6] in der Modifikation von Joy und Luo [7]. Sekundärelektronen (SE) werden durch ein einfaches exponentielles, parametrisches Model generiert und transportiert, dabei werden zwei materialabhängige Parameter genutzt, eine mittlere SE-Anregungsenergie und eine mittlere Austrittslänge. Entstandene Sekundärelektronen erhalten denselben Zeitindex wie das sie erzeugende Primärelektron.

In Abbildung 5 sind die Bahnkurven von 100 Primärelektronen bei $10 \mathrm{keV}$ in einer $\mathrm{SiO}_{2}$-Schicht berechnet in $v R E M$ in einem Ausgabefenster von MATLAB dargestellt.

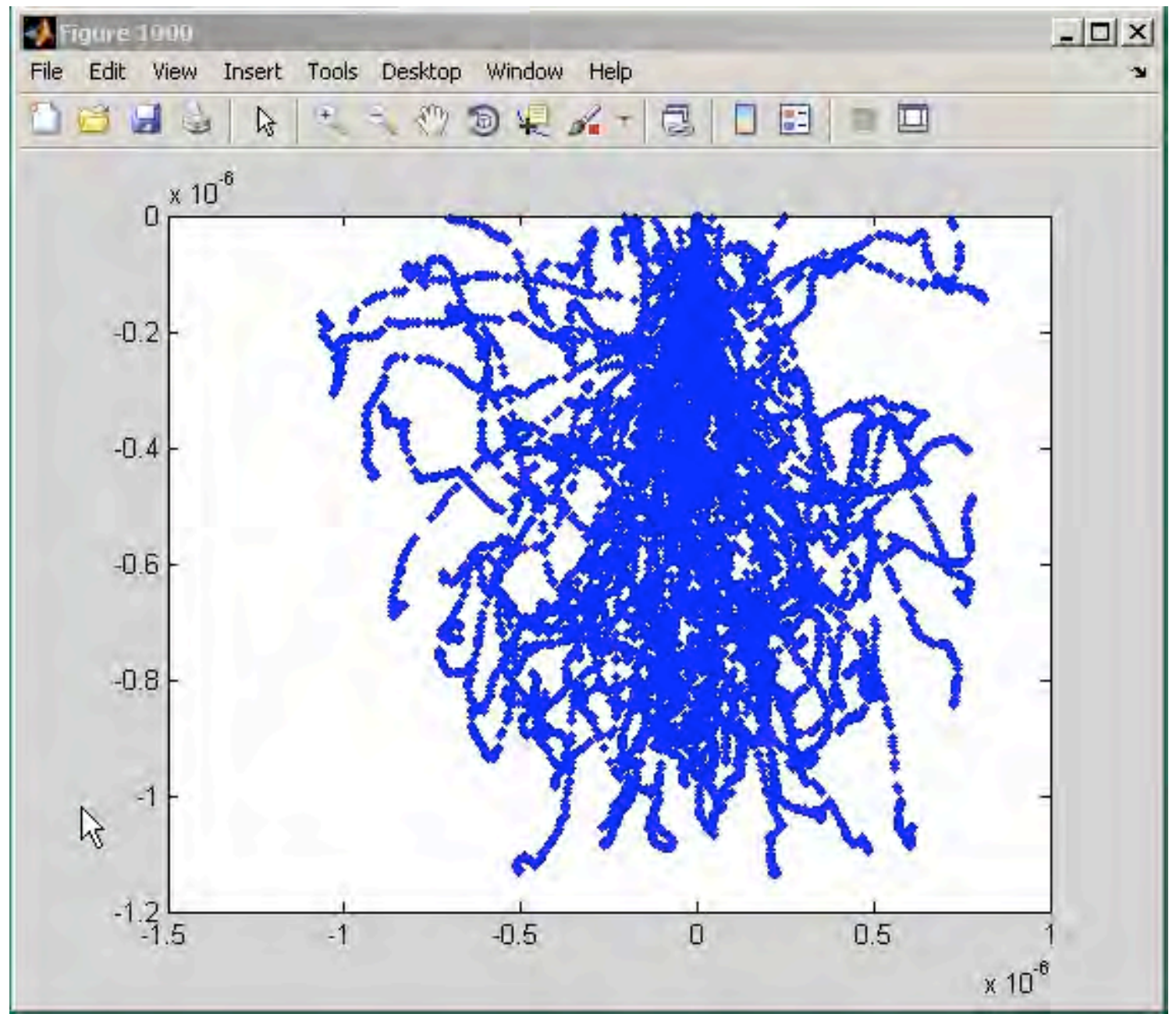

Abbildung 5: Bahnkurven von Primärelektronen berechnet von vREM 


\section{Detektion und Bildgenerierung}

Im Detektormodul werden bislang nur rudimentär die aus der Probe austretenden Elektronen, die in einen durch Parameter definierten Raumwinkelbereich abstrahlen und deren Energie innerhalb definierbarer Energiebereiche ist (Ausnahme SE) aufgrund ihres Zeitindex' einem Pixel zugeordnet.

In Zukunft werden schon vorhandene Detektoreffizienttabellen angewandt werden können, Variationen der Detektoreffizienzen (z.B. Schwankungen bei Kaskadeneffekten in den Dynoden), behandelt werden, die Detektorsignale digitalisiert werden, sowie Rauschen und Verstärkungsfaktoren der Elektronik behandelt werden. Über Kontrast- und Helligkeitseinstellungen sollen diese Signale in einen Intensitätswert für einen Pixel umgerechnet werden. Auch soll es möglich sein, Mittelungen, z.B. über mehrere Zeilen, hier einzustellen.

\section{Auswertung}

In diesem letzten Programmteil sollen einfache Auswertungs-Algorithmen umgesetzt werden, so dass direkt aus den Variationen der Parameter in den vorangehenden Programmteilen eine Unsicherheit einer in diesem Modul bestimmten Messgröße abgeleitet werden kann. Die Beobachtung des Einflusses der Auswertung auf die Bestimmung der Messgröße und Untersuchungen bezüglich der Robustheit eines Algorithmus sind weitere Einsatzmöglichkeiten dieses Programmteils.

\section{Literatur}

[1] R. Schmitt et al.: Virtuelle Messgeräte. Definition und Stand der Entwicklung. Technisches Messen 75 (2008) 5, s. 298-309.

[2] D. Gnieser, C.G. Frase, H. Bosse, R. Tutsch: MCSem - a modular Monte Carlo simulation for various applications in SEM metrology and SEM photogrammetry. Poster: 14th European Microscopy Congress (EMC 2008) 1.-5. September 2008, Aachen.

[3] L. Reimer: Scanning Electron Microscopy. Physics of Image Formation and Microanalysis. Springer Series in Optical Science Vol. 36, $4^{\text {th }}$ edition (1997).

[4] W. Häßler-Grohne, K. Hahm, W. Mirande, H. Bosse, M. Arnz: Calibration of test reticles for qualification of imaging properties of microlithographic projection lenses. Proc. SPIE 4764, $142-$ 150 (2002).

[5] F. Salvat, R. Mayol: Elastic scattering of electrons and positrons by atoms. Schödinger and Dirac partial wave analysis. Computer Physics Communications 74, 358-374 (1993).

[6] H.A. Bethe: Zur Theorie des Durchgangs schneller Korpuskularstrahlen durch Materie. Ann. Phys. 5, 325-400 (1930).

[7] D.C. Joy, S. Luo: An Empirical Stopping Power Relationship for Low-Energy Electrons. Scanning 11(4), 176-180 (1989).

\section{Danksagung}

Die Autoren bedanken sich für die Finanzierung des Projekts, über das hier berichtet wird, durch die Sachbeihilfen BO 3492/1-1 bzw. TU 135/16-1 bei der Deutschen Forschungsgemeinschaft (DFG). 